\title{
Transversus Thoracis Muscle Plane Block in Children Undergoing Open Heart Surgery via Median Sternotomy
}

\author{
Nabil A Mageed*, Ibrahim I Abd El Baser and Mohamed M Morsy \\ Department of Anesthesia and Surgical Intensive Care, Faculty of Medicine, Mansoura University, Egypt
}

*Corresponding author: Nabil A Mageed, Department of Anesthesia and Surgical Intensive Care, Mansoura University, Egypt.

Received Date: May 19, 2020

Published Date: May 27, 2020

\begin{abstract}
Sternum is the main source of pain after cardiac surgery. The use of high doses of opioid are associated with prolonged mechanical ventilation. Regional anesthetic techniques facilitate early recovery. Transversus thoracis plane (TTP) block is a newly developed analgesic technique that can provide adequate analgesia for surgeries associated with sternotomy. In pediatric cardiac surgery, the dose of local anesthetics for TTP block is not described in any of literature. In our hospital (Mansoura University Children Hospital), we perform bilateral TTP block for most of children submitted for cardiac surgery via median sternotomy in a dose of $0.2-0.3 \mathrm{~mL} / \mathrm{kg} 0.2$ to $0.25 \%$ bupivacaine on each side of sternum. We found that, the above dose provides effective perioperative analgesia without any signs of local anesthetic toxicity. The aim of this work is to highlight the analgesic efficacy of TTP block and the dose of local anesthetic in children undergoing open heart surgery via median sternotomy.
\end{abstract}

Keywords: Transversus thoracis; Pediatric cardiac; Sternotomy; Block; Surgery

\section{Introduction}

Patients undergoing open heart surgery via median sternotomy often suffer from moderate to severe pain after surgery and the classic treatment with intravenous analgesics is usually ineffective $[1,2]$. Milgrom LB et al. [2]in their prospective study, assessed postoperative pain severity in cardiac surgical patients during activities in the first postoperative 6 days. They found that, pain scores were highest during cough and movements during the first postoperative day Milgrom LB, et al. [2], inadequate management of acute postoperative pain can lead chronic persistent and distressing post-sternotomy pain in about 75 to $88 \%$ of patients [3]. Mid-sternum represents the maximum source of postoperative pain after cardiac surgery [4]. Central neuraxial techniques [5] and bilateral thoracic paravertebral block [6] have been studied to control post-sternotomy pain but the fear of hemodynamic instability, epidural hematoma and pneumothorax restricts their use in the fully anti coagulated cardiac surgical patients.

The newly developed fascial muscle plane block, transversus thoracis muscle plane (TTP) block, can provides analgesia for surgeries involving the anterior chest wall by blocking $\mathrm{T} 2$ to $\mathrm{T} 6$ anterior intercostal nerves [7-10]. Ueshima H and Kitamura A [8] were the first to demonstrate the clinical use of the TTP block in 2015 in an adult 86-year-old female patient with severely compromised cardiac function undergoing breast surgery. Ueshima $\mathrm{H}$, et al. [11] reported the effectiveness of bilateral TTP block in providing adequate postoperative analgesia in 2 adult cases submitted for cardiac surgery via median sternotomy. The current review will discuss the new advances of perioperative pain control in pediatric cardiac surgery with a special focus on the TTP block. 


\section{Pathophysiology of pain in pediatric cardiac surgery}

Pain is an unpleasant sensation following surgery, and adequate treatment is required to abolish it. Ethically, we are obligated to provide a suitable treatment to reduce the perioperative pain intensity [12]. Perioperative pain occurring during cardiac surgery have been documented to be a risk factor for increased risk of morbidity, Anand KJ, et al. [13] including cardiac dysthrryhtmia, cardiovascular and pulmonary dysfunction, hypercoagulable states, and wound sepsis. Pain causes a stress response which influence the postoperative course and patient's recovery, whatever their age. The pathophysiology and mechanism of pain during pediatric cardiac surgery have not been extensively studied as in adults [14]. In adult cardiac surgery, pain is most severe during the first postoperative day and subsides gradually over the next few days. Cardiopulmonary bypass (CPB) may worsen the severity of postoperative pain as it induces a systemic inflammatory response. In children, the severity, duration and distribution of pain are not obvious and difficult to assess in due to the presence of multiple and variable pain scores, each one is suitable only for certain pediatric age group.

\section{Perioperative control of pain and stress response in pediatric cardiac surgery}

\section{Systemic opioids}

Systemic opioids have been used as a classic and routine way for management of perioperative pain in children undergoing cardiac surgery through median sternotomy. Each child responds to pain by a different way than the other, as pain is subjective, however the treatment of pain is usually based on standardized protocols for all patients and not specific for certain individual [15].

In pediatric cardiac surgery, the used dose of opioids has been modified over time. The use of high doses of opioids during surgery have been shown to decrease the stress response [13]. The disadvantages of the use of high dose opioid include, prolonged mechanical ventilation, tolerance, and the side effects related to opioid administration (nausea and vomiting, sedation, gastro-intestinal dysfunction, and immunosuppression). Five different dose regimens of fentanyl were studied in a prospective randomized study to evaluate the effect of different doses of fentanyl on the stress response during the pre by pass period in pediatric cardiac surgery [16]. Fentanyl in a dose of $25-50 \mu \mathrm{g} / \mathrm{kg}$ (as part of a balanced anesthetic) was recommended, as a part of balanced anesthesia, to suppress the hemodynamic and metabolic stress response. Recently, lower doses of opioids are often used to facilitate early recovery and extubation, but the use of such doses don't guarantee complete blunting the stress response. Inadequate control of perioperative pain in children with redo-cardiac may be associated with development of chronic post-sternotomy pain, although this is less frequent than in adults after redo sternotomy.

\section{Local anesthetic techniques}

The use of local anesthetic techniques can avoid the need for high doses of opioids. These techniques interfere with the afferent impulses from the accompanying nerves, thus minimizing or abolishing the need for opioids [17]. Regional anesthetic techniques have been shown to reduce postoperative pain up to 24 hours in children undergoing cardiac surgery [18]. Central neuraxial anesthetic techniques including, caudal, Sendasgupta C, et al. [19] epidural, Mehta Y and Arora D [5] and spinal, Humphreys N, et al. [20] have been used since the 1980s but their use is limited, unlike their use in noncardiac surgery, due to the possible risk epidural hematoma in the fully anti coagulated patients. Monahan A, et al. [21] recommended the use of superficial inter-fascial muscle plane blocks to provide adequate perioperative analgesia. To relieve perioperative pain originating from sternotomy incision, it is essential to block the second to sixth anterior intercostal nerves that innervate the sternum [1].

\section{Transversus thoracic muscle Plane (TTP) block}

Transversus thoracic muscle plane (TTP) block was first reported in 2015 for providing adequate analgesia in conjunction with pectoralis nerve (PECS) II block in breast surgery. TTP block anesthetize the anterior intercostal nerves (T2-6) [8, 10]. Bilateral TTP block for perioperative pain control in adult cardiac surgery has recently been reported [22].

TTP block can be done by injection of local anesthetics in the fascial plane between the internal intercostal and transversus thoracis muscles. In a cadaveric study guided by ultrasound, the TTP block was found to cover the T2-T6 anterior intercostal nerves [7]. The anterior branches of these intercostal nerves are responsible for the sensory innervation of the internal mammary area, indicating that, this new fascial plane block can provide analgesia for surgery of the anterior chest wall. This was followed by the study of Ueshima et al. who reported the successful use of TTP block in combination with bilateral pectoral nerve blocks (Pecs II) for bilateral breast cancer resections without the need for general anesthesia [9].

\section{Technique}

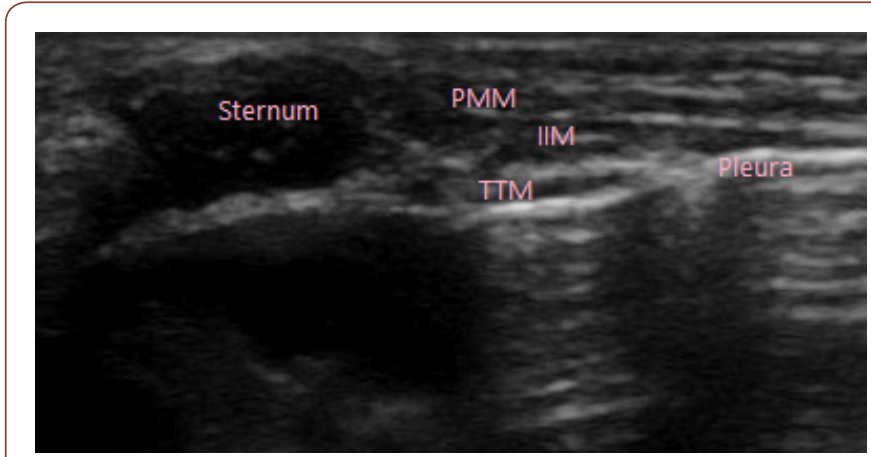

Figure 1: PMM: Pectoralis Major Muscle; IIM: Internal Intercostal Muscle; TTM: Transversus Thoracis Muscle.

TTP block is performed by the use of high frequency ultrasound linear probe. Initially, the ultrasound probe is placed alongside of the sternum at about $1 \mathrm{~cm}$ from sternal border to count intercostal spaces. The ultrasound probe is rotated by 90 degree to be 
positioned in the space between the 4 th and 5 th rib. The fascial plane between internal intercostal and the transversus thoracis muscles is identified (Figure 1). A 22-gauge, 80 -mm short bevel needle is used and advanced in in-plane manner until the tip of the needle is located in the fascial plane between the internal intercostal and transversus thoracis muscles. Local anesthetic is administered after exclusion of intravascular and intrapleural placement. Downward displacement of the pleura (Figure 2) is a good indicator of proper injection into the TTP. The patient is continuously monitored for at least 20 minutes for any manifestations of local anesthetics toxicity, allergic reaction and hemodynamic instability. Regarding the best intercostal space for performance of TTP block, a study involving 10 healthy volunteers found that, injection of local anesthetic at the T4-T5 intercostal space was accompanied with improvement of the spread of local anesthetic compared with injection at T3-T4 [23] (Figure 1)(Figure 2)

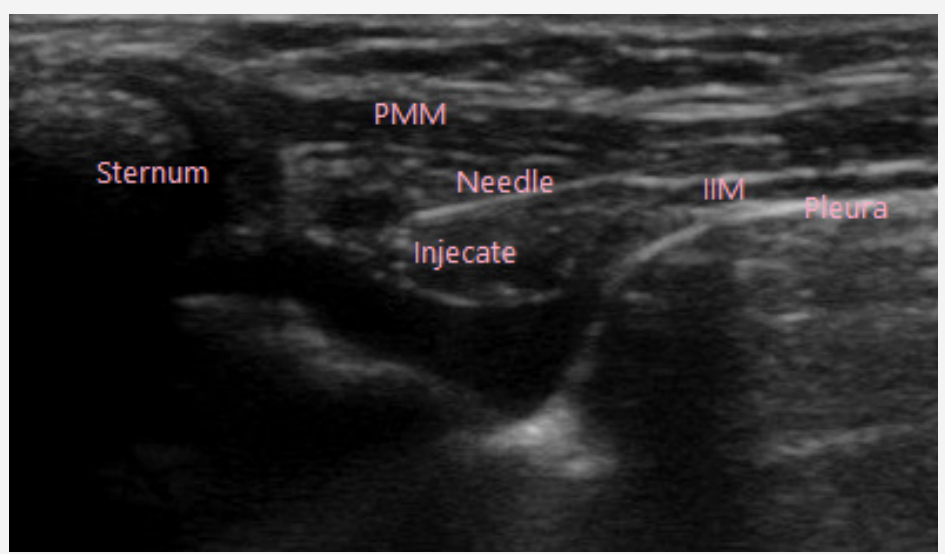

Figure 2: PMM: Pectoralis Major Muscle; IIM: Internal Intercostal Muscle; TTM: Transversus Thoracis Muscle.

\section{The pediatric dose of local anesthetics for TTP block}

The adequate dose of local anesthetics for TTP in pediatric is not described in any of literatures. In adult cardiac surgery, the reported dose of ropivacaine was $20 \mathrm{ml} 0.3 \%$ or $0.5 \%$ on each side of the sternum [24]. In our hospital (Mansoura University Children Hospital) TTP block is performed for most of children submitted for cardiac surgery via median sternotomy and is used as a part of balanced anesthesia to reduce the total perioperative fentanyl requirements, and thus, allowing early recovery. We use 0.3 to 0.4 $\mathrm{ml} / \mathrm{kg}$ bupivacaine 0.2 to $0.25 \%$ on each side of the sternum, such dose is associated with adequate perioperative analgesia without any manifestations of local anesthetic toxicity.

\section{Complications}

TTP block related complications include vascular injury, local anesthetic toxicity, pneumothorax, failed block, and anaphylactic shock. The structures adjacent to the puncture site are vulnerable to needle traumatic injury and include the internal mammary vessels, pleura, pericardium, and intercostal vessels. Ueshima H, et al [25] followed up 299 patients in whom the TTP blocks were performed and found that only 2 patients developed mild infection in the skin at the puncture site. Further studies with larger sample sizes are required to estimate the incidence of these rare complications.

\section{Conclusion}

Transversus thoracis plane block is a newly developed analgesic technique that can provide adequate perioperative analgesia in pediatric cardiac surgery. According to our experience, the adequate dose of bupivacaine in pediatric is $0.3-0.4 \mathrm{ml} / \mathrm{kg}$ bupivacaine 0.2 $0.25 \%$ on each side of the sternum.

\section{Acknowledgment}

None

\section{Conflict of Interest}

No conflict of interest.

\section{References}

1. Mazzeffi M, Khelemsky Y (2011) Poststernotomy pain: a clinical review. J Cardiothorac Vasc Anesth 25:1163-1178.

2. Milgrom LB, Brooks JA, Qi R, Bunnell K, Wuestfeld S, et al. (2004) Pain levels experienced with activities after cardiac surgery. Am J Crit Care 13: 116-125.

3. Kamalipour H, Vafaei A, Parviz Kazemi A, Khademi S (2014) Comparing the prevalence of chronic pain after sternotomy in patients undergoing coronary artery bypass grafting using the internal mammary artery and other open heart surgeries. Anesth Pain Med 4: e17969.

4. Mueller XM, Tinguely F, Tevaearai HT, Revelly JP, Chioléro R, et al. (2000) Pain location, distribution, and intensity after cardiac surgery. Chest 118: 391-396.

5. Mehta Y, Arora D (2014) Benefits and risks of epidural analgesia in cardiac surgery. J Cardiothorac Vasc Anesth 28: 1057-1063.

6. El Bendary HM, El Baser IA (2015) Bilateral single bupivacaine injection ultrasound-guided paravertebral block facilitates early extubation and reduces perioperative opioids requirements in on-pump pediatric cardiac surgery. Ain-Shams J Anesthesiol 8: 287-293.

7. Ueshima H, Takeda Y, Ishikawa S, Otake H (2015) Ultrasound-guided transversus thoracic muscle plane block: a cadaveric study of the spread of injectate. J Clin Anesth 27: 696.

8. Ueshima H, Kitamura A (2015) Blocking of multiple anterior branches of intercostal nerves (Th2-6) using a Transversus thoracic muscle plane block. Reg Anesth Pain Med 40(4): 388.

9. Ueshima H, Kitamura A (2015) Clinical experiences of ultrasoundguided transversus thoracic muscle plane block: a clinical experience. J Clin Anesth 27: 428-429. 
10. Ueshima H, Otake (2017) Addition of transversus thoracic muscle plane block to pectoral nerves block provides more effective perioperative pain relief than pectoral nerves block alone for breast cancer surgery. $\mathrm{Br}$ J Anaesth 118: 439-443.

11. Ueshima H, Hara E, Marui T, et al. (2016) The ultrasound-guided transversus thoracic muscle plane block is effective for the median sternotomy. J Clin Anesth 29.

12. Carvalho AS, Martins PS, Jácomo A, Magalhães S, Araújo J et al. (2018) Ethical decision making in pain management: a conceptual framework. J Pain Res 15: 967-976.

13. Anand KJ, Hansen DD, Hickey PR (1990) Hormonal-metabolic stress responses in neonates undergoing cardiac surgery. Anesthesiology 73 661-670.

14.Zubrzycki M, Liebold A, Skrabal C, Reinelt H, Ziegler M (2018) Assessment and pathophysiology of pain in cardiac surgery. J Pain Res 24: 1599-1611.

15. Olkkola KT, Hamunen K, Maunuksela EL (1995) Clinical pharmacokinetics and pharmacodynamics of opioid analgesics in infants and children. Clin Pharmacokinet 28: 385-404.

16. Duncan HP, Cloote A, Weir PM, Jenkins I, Murphy PJ, et al. (2000) Reducing stress responses in the pre-bypass phase of open heart surgery in infants and young children: a comparison of different fentanyl doses. Br J Anesth 84: 556-564.

17. Wolf AR (2012) Effects of regional analgesia on stress responses to pediatric surgery. Pediatr Anesth 22: 19-24.

18. Monahan A, Guay J, Hajduk J, Suresh S (2019) Regional analgesia added to general anesthesia compared with general anesthesia plus systemic analgesia for cardiac surgery in children: a systematic review and meta-analysis of randomized clinical trials. Anesth Analg 128: 130-136.

19. Sendasgupta C, Makhija N, Kiran U, Choudhary SK, Lakshmy R, et al. (2009) Caudal epidural sufentanil and bupivacaine decreases stress response in pediatric cardiac surgery. Ann Card Anesth 12: 27-33.

20. Humphreys N, Bays SM, Parry AJ, Pawade A, Heyderman RS, et al. (2005) Spinal anesthesia with an indwelling catheter reduces the stress response in pediatric open heart surgery. Anesthesiology 103: 1113-1120.

21. Monahan A, Guay J, Hajduk J, Suresh S (2019) Regional analgesia added to general anesthesia compared with general anesthesia plus systemic analgesia for cardiac surgery in children: a systematic review and metaanalysis of randomized clinical trials. Anesth Analg 128: 130-136.

22. Ueshima H, Otake H (2016) Continuous transversus thoracic muscle plane block is effective for the median sternotomy. J Clin Anesth 37: 174.

23. Ueshima $H$, Otake $H$ (2016) Where is an appropriate injection point for an ultrasound-guided transversus thoracic muscle plane block? J Clin Anesth 33: 190-191.

24. Fujii S, Roche M, Jones PM, Vissa D, Bainbridge D, et al. (2019) Transversus thoracis muscle plane block in cardiac surgery: a pilot feasibility study. Reg Anesth Pain Med 44: 556-560.

25. Ueshima H, Otake H (2017) "Ultrasound-guided transversus thoracic muscle plane block: Complication in 299 consecutive cases. J Clin Anesth 41: 60 . 